Please quote as: Prinz, A.; Menschner, P. \& Leimeister, J. M. (2012): Electronic Data Capture in HealthCare - NFC as Easy Way for Self reported Health Status Information. In: Health Policy and Technology, Ausgabe/Number: 3, Vol. 1, Erscheinungsjahr/Year: 2012. 
Provided for non-commercial research and education use. Not for reproduction, distribution or commercial use.

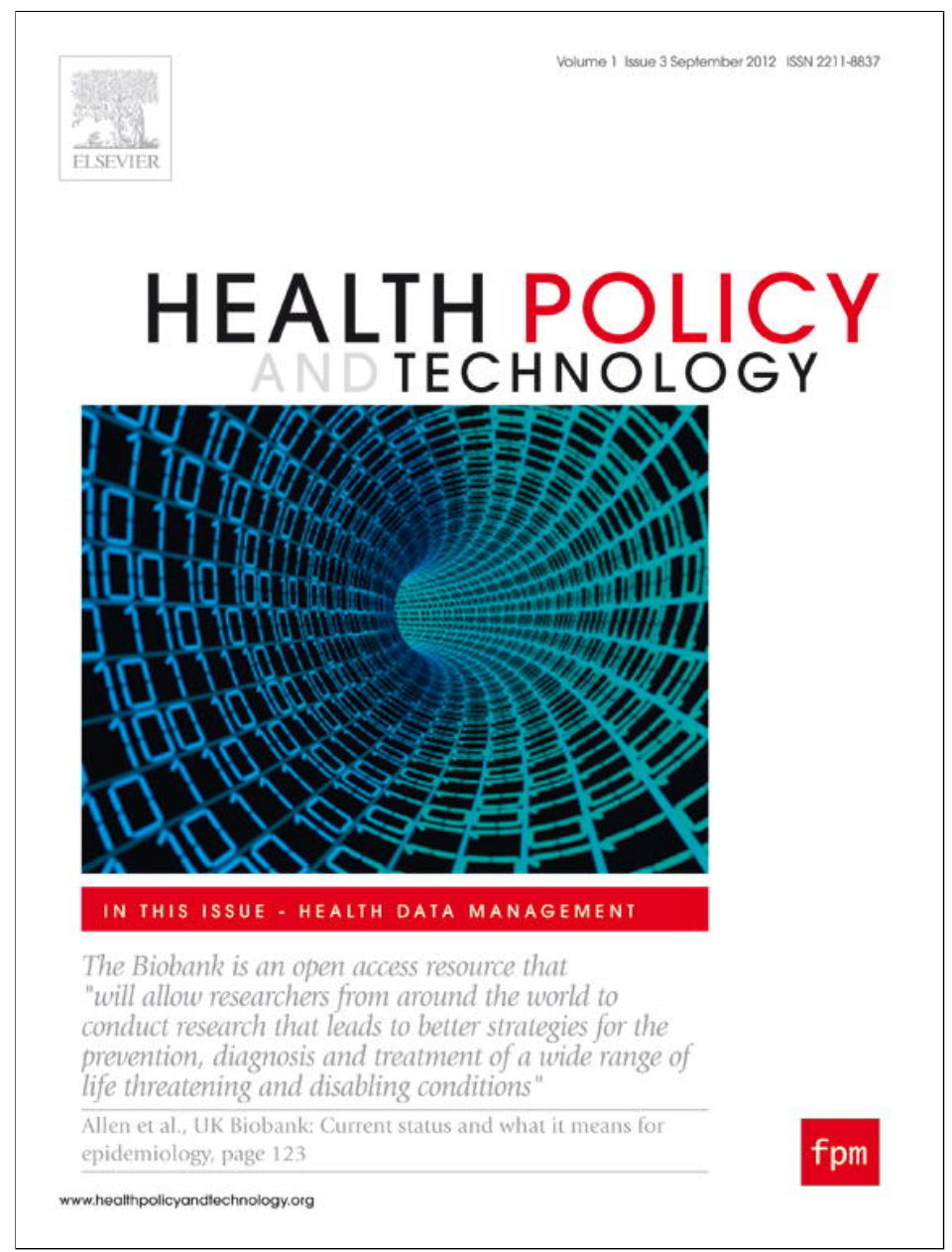

This article appeared in a journal published by Elsevier. The attached copy is furnished to the author for internal non-commercial research and education use, including for instruction at the authors institution and sharing with colleagues.

Other uses, including reproduction and distribution, or selling or licensing copies, or posting to personal, institutional or third party websites are prohibited.

In most cases authors are permitted to post their version of the article (e.g. in Word or Tex form) to their personal website or institutional repository. Authors requiring further information regarding Elsevier's archiving and manuscript policies are encouraged to visit:

http://www.elsevier.com/copyright 


\title{
Electronic data capture in healthcare-NFC as easy way for self-reported health status information
}

\author{
Andreas Prinz*, Philipp Menschner, Jan Marco Leimeister
}

\author{
Information Systems, Kassel University, Pfannkuchstraße 1, 34121 Kassel, Germany
}

Available online 10 August 2012

\begin{abstract}
We present an electronic data capture (EDC) system based on Near Field Communication (NFC). NFC is an easy way for self-reporting of health status information. As traditional paper-based questionnaires are time- and cost-consuming and may be affected by low patient compliance, our EDC system allows patient monitoring and electronic data acquisition directly from the patient's home. It enables real time representation and analysis of patient data and thus allows direct medical intervention by physicians. The results of a field test indicate that NFC is almost as simple as filling out a paper-based questionnaire. During the study patients used the prototype autonomously and with minimal errors. Further, NFC technology was perceived as very intuitive and the information quality of each patient's health status could be improved. Based on the findings we derive recommendations for future research and applications of NFC based electronic data capture systems.
\end{abstract}

(c) 2012 Fellowship of Postgraduate Medicine. Published by Elsevier Ltd. All rights reserved.

\section{Introduction}

Patients suffering from chronic diseases, such as motor neuron disease, dementia, Parkinson's disease or multiple sclerosis, often have to cope with limitations and a reduced quality of life $[38,22]$. In order to guarantee best possible treatment procedures, physicians need up-to-date information about their patients' actual state and well-being. Yet, it is well recognized that capturing, archiving, analyzing and interpreting health status information involves logistic challenges [39]. For patients living at home, this implies an even greater challenge, as contemporaneous information, which can be used for therapeutic decision-making, is not available. The insufficient information logistics amongst physicians, patients and nursing staff could be improved by using mobile networks and information systems for electronic data capture

\footnotetext{
*Corresponding author. Tel.: +495618046062.

E-mail address: prinz@uni-kassel.de (A. Prinz).
}

(EDC) of health status information. As mobile devices have achieved a high level of public acceptance, they are increasingly considered to be integrated in health care $[21,40]$, as they allow flexible, location-independent monitoring of the patients. Additionally, costs can be reduced as patients take over data acquisition tasks.

In this paper, we describe the development and evaluation of a mobile Near Field Communication (NFC)-based electronic data capture (EDC) system for patient self-reported questionnaires. NFC, is a short-range high frequency wireless communication technology, based on the frequency of $13.56 \mathrm{MHz}$, which enables the exchange of data between devices within $10 \mathrm{~cm}$ distance $[8,37]$. The objective of our work was to provide a simple, effective and efficient self-reporting system, which would allow patients a cost-efficient and easy-to-handle reporting system of their current condition. We identified a scenario that can benefit from applying EDC techniques, both from an economic perspective as well as having an impact on the patients' health status and quality of life. These are rating scales and disease progress questionnaires. 


\section{Related work}

In health care, the provision of appropriate treatment often depends on self-reported states. Over the last decades, there has been a steady progress in the development of measurement techniques for all kinds of diseases. A wide variety of rating scales is used to determine the state of patients, ranging from impairment scales to health-related quality of life instruments (e.g., $[18,11,38])$. Studies have shown their importance in measuring the impact of different diseases and their treatment on patients [12]. The effectiveness of patient self-rating for different kinds of diseases has been evaluated [34], showing that validity and reliability are of a sufficient level in order to serve as a basis for treatment decision-making. Follow-up costs of inadequate treatment are hard to measure, and the negative impact on patients' quality of life is manifest.

Health status information has traditionally been captured using paper-based questionnaires. However, these are timeand cost-consuming, and can suffer from contaminating effects, such as time-delay or inaccuracy due to disregard. Electronic-based questionnaires overcome these deficits by allowing to aggregate and calculate data, which potentially reduces the time to acquire data and can hence increase the patient's compliance [26]. Further, this data can instantly be compiled and be available for physicians and clinical personnel in almost real time. This provides indications for possible medical interventions, even in-between appointments [36].

There are studies on various occurrences of electronic data capture (EDC) $[3,31,27,6,19,4]$. The application of NFC technology to provide EDC is, however, comparatively novel and not widely implemented. NFC makes many tasks easier and more convenient by providing access to systems and services simply by touch. Morak et al. [25] use NFC as a selfmanagement process for monitoring heart failure patients and Iglesias et al. [14] describe an NFC-based health monitoring system to improve quality of life for elderly patients. Patients transmit health related data to a central database by touching medical devices with a mobile telephone. Physicians or nurses can view the entire data and guide the patient to the best possible health status. Bravo et al. [5] use NFC for supporting nurse activities in an Alzheimer's day centre. NFC-based nutrition management for elderly patients is suggested by Prinz et al. [29]. In a clinical context, NFC is used by various researchers. Lahtela et al. [20] have developed an NFC-based solution to avoid medication errors in hospitals. As an additional path of medical data acquisition, Fikry et al. [9] and Morak et al. [24] describe different NFC-based solutions which allow physicians or nurses to collect data by easily touching medical devices with a mobile phone. Yet, none of these studies focus on usability for patients suffering from impaired fine motor skills.

\section{Development and research approach}

For the specific use case we worked in cooperation with a German hospital which is specialized on amyotrophic lateral sclerosis (ALS), a form of motor neuron disease. ALS is a progressive, fatal, neurodegenerative disease caused by the degeneration of motor neurons, the nerve cells in the central nervous system that control voluntary muscle movement. Death usually occurs within two to five years of diagnosis. In particular, ALS patients suffer from progressive paralysis, so they need to be provided with an easy to handle system. As patients attribute an important role to the interactive applications interface design, the overall process of our approach to user-centered design (UCD) is based on evaluating the usability of inSERT in small iterative steps.

\section{Design goals}

The design goals have been identified in discussions and observations with physicians and patients and formed the basis for developing our solution idea for an EDC system. In this specific case we had several challenges. As continuous tracking and surveillance of health status did not exist thus far, means for patients to report on their current state had to be integrated. Another particularity was the progressive paralysis of patients. Due to impaired fine motor skills, it was difficult for these patients to use commonly available user interfaces such as mouse or multi touch devices. Our concept of inSERT, an easy-to-use EDC system for patients with impaired fine motor skills, is based on the following main goals:

- Increase the usability (a) ease of use and (b) perceived usefulness: a major problem in mobile user-interface (UI) design practice is that current approaches cannot directly be applied to mobile phones. According to Subramanya and Yi [35], in general, the UI should be intuitive and easy to use. The usability is important for creating applications which are easy to use and which fulfil the needs of the targeted user-group. Ease of use and perceived usefulness derive directly from the TAM [7].

- Increase intention to use: the dimension intention to use is a predictor of future usage, it derives directly from the TAM [7].

- Increase confirmation: the questions about confirmation are an important dimension for explaining IS continuance intention [2].

- Providing a very efficient and intuitive way of interaction suited to patients with impaired fine motor skills: it is important that patients with impaired fine motor skills are able to use the system. Otherwise, it is no guarantee that the data will be entered reliably.

- Improve information quality: are the results useful to serve as medical decision-making basis and facilitate the additional information of physician daily work?

\section{Research approach}

We used a design science approach $[16,13]$ which aims to develop solutions to organizational problems through design and evaluation of novel artefacts. The design process generally consists of analyses, design, implementation and evaluation of an artefact $[33,13]$, in our case the prototype 
inSERT. As users generally had no prior experience with mobile EDC services, and user involvement in system development is still an acknowledged success factor, we adopted the AAL service design (AALSDA) approach by Menschner et al. [23] (Figure 1).

We began with an in-depth analysis of treatment and care processes of patients suffering from ALS. Background information on the problem was obtained through a review of relevant literature, case studies, interviews, questionnaires, observations and document analyses [41]. Based on the findings from the analyses and our design goals, we developed a design concept and a low fidelity prototype which was then evaluated in focus groups as well as in workshops [28]. We then developed a fully functional proofof-concept prototype which was tested in a long-term field study in a German hospital.

\section{The design of the inSERT prototype}

A mobile phone application that allows the reading of NFCtags on a smart poster with NFC-enabled mobile phones is used. By touching a tag with an NFC-enabled mobile phone, this information can be read and displayed to the user [10]. The transmitted and aggregated data is processed and stored in the central unit of the inSERT system and can be analyzed by physicians and nurses.

\section{Smart poster and mobile application}

NFC solutions can outperform iPhone and PC-solutions as well as a paper-based protocol with respect to pragmatic and hedonistic aspects [30]. The easy to learn touch metaphor of NFC interaction hence makes NFC an interesting technology, particularly for our target group.

Figure 2 shows the self-rating questionnaire smart poster of the well-established ALS-functional rating scale [18]. The smart poster contains 12 questions (three sections to bulbar symptoms, motor activity and respiratory dysfunction) with five possible answers in each case [17]. The poster has images printed in front and NFC-tags glued to the back.

To start the application, a touch on the "start" icon or interaction field is sufficient. After the application is launched, the patient has to touch one of the 12 questions.
As shown in Figure 3, patients have to follow four simple steps to answer the question via inSERT.

Once contact is made, the mobile device gives audible, haptic and visual feedback to the user. For audible feedback, the title of the chosen question is spoken; for haptic feedback, the phone vibrates; and for visual feedback, the chosen headline of the question appears on the display. After each question the patient has to respond to a fivepoint Likert scale (0-4). Once the answering process for the chosen question is completed, the application reminds the patient to answer the next question. Again, the mobile device confirms the transmission of data with audible, haptic and visual feedback.

The icon of the interaction field is based on generic NFC tags [1]. To help patients navigate to the interactive areas, the inner circle is coloured red. The size of the interaction field of $35 \times 35 \mathrm{~mm}$ is dependent on the used passive NFC tags. The client is implemented as a Java J2ME midlet which controls the NFC-reader device on a Nokia 6212 [15].

\section{Physician dashboard}

Further, a physician dashboard has been implemented through which physicians can extract relevant information about their patients. In order for the information to be available anytime and anywhere, a web portal has been implemented. The dashboard is structured as follows: on one side there is a category 'patients' providing patient relevant data. On the opposite side there is a category 'mobile devices' referring to the mobile phone data handed out. In the 'patients' category, physicians get an overview representation of the patient data, where they can see information about all participating patients. This is where patients can be added or deleted. In the hospital, the transmitted data is merged with the patient data. As data security was an issue, we separated master data from transaction data.

Figure 4 depicts the answers of the last questionnaire entries. The diagram clearly illustrates the changes in the assessment of patients over their period of scoring. In addition to the access for physicians, the dashboard contains a special administrator access. This area allows assigning of access privileges to specific people in different roles. It is thus possible to define the physicians responsible for patients or further

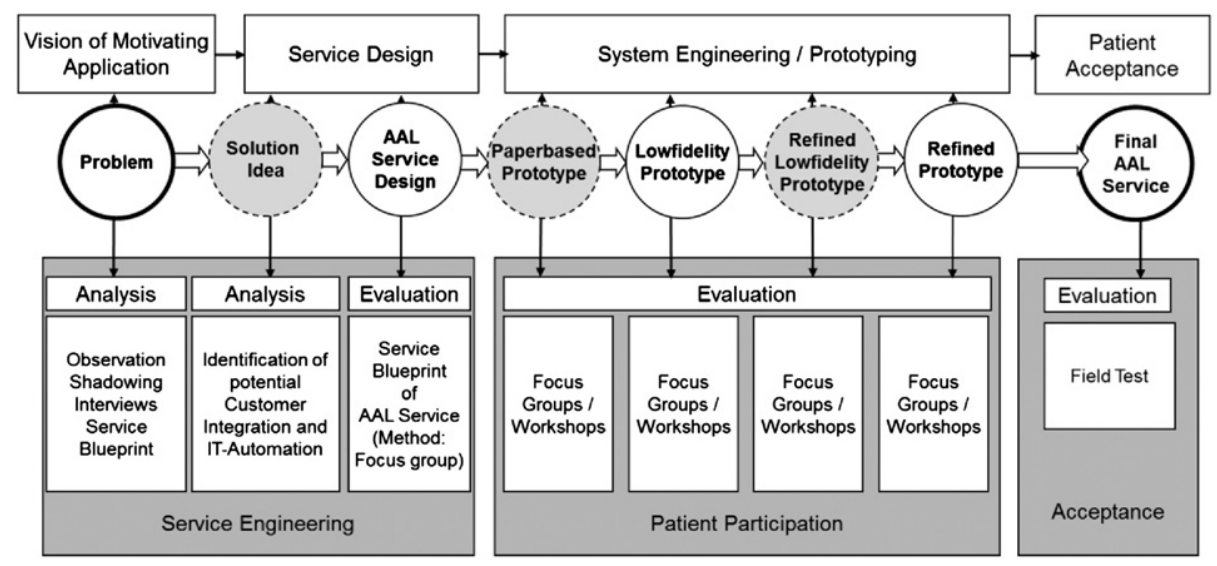

Figure 1 AALSDA approach [23]. 


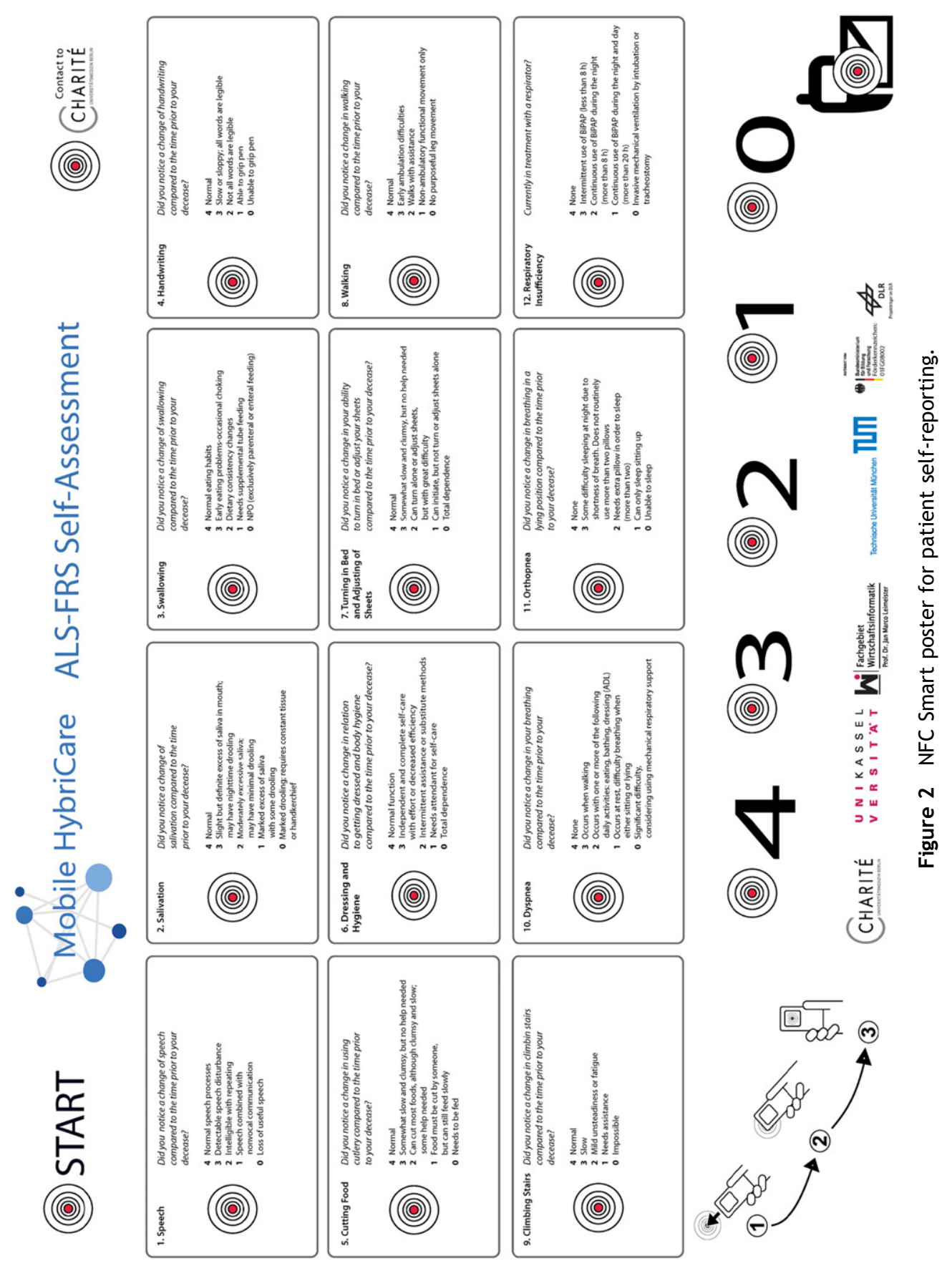


1. Touch the "start" icon to launch the application installed on the phone.

2. Touch one of the twelve questions.

3. Touch again on the rating scale to answer the question.
3. (Step two and three is repeated twelve times)

4. Touch on the "senden" [send] tag and confirm it with a second touch to send the data to the physician.

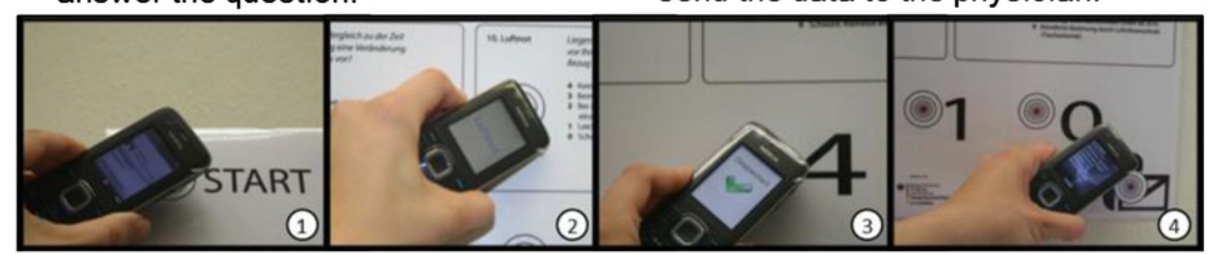

Figure 3 Rating process of inSERT.

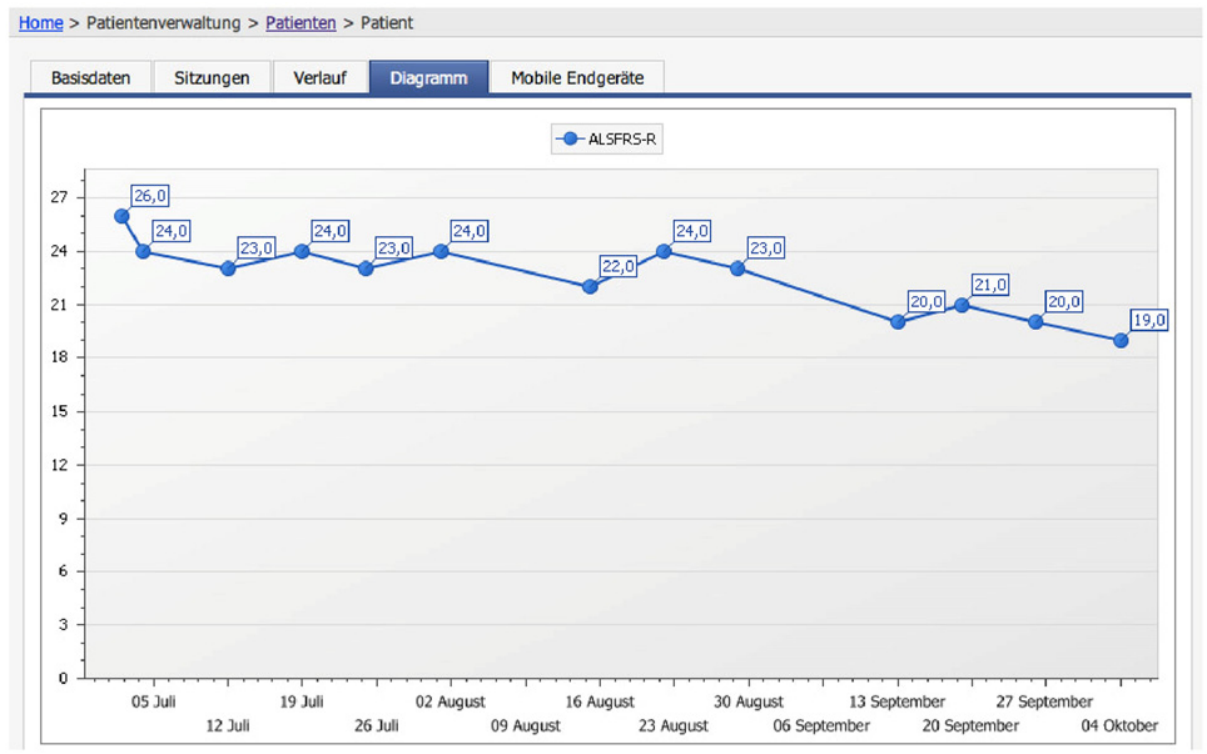

Figure 4 Physicians dashboard: patient scoring data.

administrators can be allotted. The administrator access also allows adding additional questionnaires. Therefore, a part from the existing system of evaluating a questionnaire concerning motor diseases, further smart poster and tele-medical applications can be appended following similar principles.

\section{Evaluation setting}

The prototype was evaluated in a field study at patients' home with 18 patients' (amongst them 7 female), ranging in age from 55 to 70 years, of a German hospital over the course of 12 weeks. First, the physicians received a $45 \mathrm{~min}$ hands-on training to use and explain the system. After that, the physicians gave a training session to the patients, so that these were able to use the system at home. We had two drop outs, one of them died during the field test, the other one had usage problems - the power on/off button of the mobile phone was too small.

The objectives of this field study were (1) a test of the technical feasibility and (2) an evaluation of the practicability, according to our design goals.

- Test of the technical feasibility of the NFC-based electronic data capture system for patients with impaired fine motor skills: the relevant issue in this case was to find out whether the patients are able to use the NFCbased mobile phones with the smart poster with an acceptable error rate.

- Evaluations of the practicability of the electronic data capture system: in order for the proposed system to be beneficial in capturing patient data, the system would have to be able to run stable at patients' home without attendance, aside from assembly and disassembly. Furthermore, it needs to be assessed whether the prototype application was capable of gathering patient data at patients' home.

Every participant received an NFC-enabled mobile phone and a smart poster containing 12 questions, as described earlier. The patient had to rate him or herself once a week on a fixed day. After 12 weeks, the patients fill in a questionnaire (regarding usability, intention to use, confirmation and interaction) with a 7-point likert scale and post it together with the mobile phone and smart poster in a prepaid package to us. We received all distributed mobile phones and smart posters, but only 13 of 18 questionnaires. 


\section{Evaluation results}

The results of the evaluation are structured using the design goals: (1) increasing usability, (2) intention to use, (3) confirmation, (4) providing a very efficient and intuitive way of interaction suited to patients with impaired fine motor skills and (5) improving information quality. Interpretations are accompanied by information gathered during telephone interviews with patients and physicians. We conducted evaluations on a technological (technical feasibility), user (practicability) and physician's basis.

\section{Overall impression}

The evaluation shows that the prototype has great potential to improve the information logistics problem between physicians, home care nurses, and patients with impairment of fine motor skills. Through the active participation and integration of patients, better documentation and an improved basis for medical treatment and nursing care could be achieved. Further, through an intensified integration, patients were enabled to better understand their medical condition and could participate more actively in the treatment and consultation processes. Our results indicate that customer (in our case patients) integration can lead to modifications for an enhanced patient-physician relationship enabled by improved patient information and autonomy. We also surmise that the prototype can improve productivity of medical processes not only by accounting for improved standards of medical care and quality of life, but also by being more cost efficient. "The use of inSERT is almost as simple as filling in a paper-based questionnaire!" One of the patients died during the field study; with the help of the captured data this could have been anticipated by a physician shortly before. Generally, a healthy person starts with an ALSFRS base score of 48. Our patients had an average score of 31.15 points, starting with an average of 33.72 and finished after 12 weeks with an average of 30.61. If the score is less than 20 points, the patient will normally die within the next few weeks. The mentioned patient started with a score of 24 and fell below the threshold of 20 within a few weeks. Furthermore, we received requests from three patients whether they could continue to use the system even after the end of the field test. The physicians' feedback was positive and there were no complaints. The subjectively assessed values have been regarded as realistic by the physicians. They checked the transmitted data with values before and after the field test. "With the help of the transmitted data, we have an accurate data base on the individual patient. Before we used the system, we have examined the patient every three to six months and in the meantime we have received no information about the patients' health status. The additional data allows us to adjust the treatment if the patient's condition changes."

\section{Evaluation according to design goals}

The results of the evaluation are structured according to the five design goals.

- Increase the usability (1a) ease of use and (1b) perceived usefulness: the overall feedback of the user acceptance is very high. Thus, it can be assumed that the operation with the prototype is easy to learn and comprehend. The patients scored the used EDC system as a system which is easy to use for them. The interaction with the system did not require a lot of mental effort. The perceived usefulness has been rated as high, with the use of inSERT the documentation frequency of disease progression would increase. Furthermore, the usefulness for documenting the disease progression has been rated high. The patients could use the system without the help of relatives or nursing staff.

- Increase intention to use (2): as we can see in the evaluation results, the intention to use has been rated high. The results match the statements of telephone interviews and showed that some patients are interested in keeping the system in use after the field study.

- Increase confirmation (3): the confirmation of the system has been rated high. The functionalities of the system were better than the patients had expected. Overall, the expectations of the system were fulfilled. In summary, we could say that all the basic features were present and the patients did not miss any main functionality.

- Providing a very efficient and intuitive way of interaction suited to patients with impaired fine motor skills (4): with inSERT we can show, that the system provides a very efficient and intuitive way of interaction suited to patients with impaired fine motor skills. The patients have rated the use of NFC technology very high, the use of buttons to interact with the system scored worse. The vibration and acoustic statement of the mobile phone by touching an interactive field was a helpful functionality.

- Improve information quality (5): As a test of the technical feasibility of the systems, the mentioned field study was a great success. We received 10.7778 (average) records per patients with a standard deviation of 3.6871. A total of 2328 values were transmitted, with an error rate of $0.34 \%$ ( 8 data points), which have been caused by a double assessment or missing data point. The average assessment interval was 176:40:24 $\mathrm{h}$ with a standard deviation of $64: 23: 41 \mathrm{~h}$. With the collected data, the physicians have a weekly picture of the patient's condition. Before they used the system, they generated this data only when the patients came to the hospital during regular appointments every three to six months.

The prototype further revealed some design principles for NFC-based EDC in general. These include the importance of feedback (audible, visible and haptic), a comprehensible and coherent poster structure and measurements to ensure data security. Noted was the appreciation of the automated reminders and warnings. Patient responses in our study showed that this gave them a feeling of being cared for. We assume that the prototype system could be applied to other contexts or groups of patients in health care, such as elderly people who face similar difficulties in handling electronic devices.

\section{Conclusion and future work}

Because of the rarity of the disease, we only had a very small number of cases and a generalization is not always 
possible. This research paper describes the development, implementation and evaluation of an application that allows patients with impaired fine motor skills to assess their health status, based on an established rating scale. We therefore adapted NFC technology to a quality of life questionnaire. We focused on engineering an easy to use interaction. Our evaluation showed that patients with impaired fine motor skills accepted inSERT as a system that is easy to use and effective for reporting the patient's current condition. Through the active participation and integration of patients, improved documentation as well as an improved basis for medical treatment and nursing care could be achieved. Further, through an intensified integration, patients gained better understanding of their medical condition and were able to participate more actively and cooperate in the treatment and consultation processes. The example of inSERT shows the potentials and possibilities that technical support systems can offer to patients. The latter exemplary extension is based upon literature documenting that the completion of specific questionnaires enable improved forecasts of disease progression [18]. The recent integration of sensors (e.g., motion sensor) and additional technical devices (e.g., global positioning system (GPS) devices) in new generations of mobile phones additionally enable completely novel medical applications [32], which could extend our work. To conclude, the use of mobile services and information technology can have a great impact on medical processes and services. Improvements of communication and interaction processes have a positive influence on the quality of patients' lives. A modified patient-physician relationship, as a result of improved patient information and autonomy, offers new possibilities for the design of novel medical services which are only made possible by modern technical support systems.

\section{References}

[1] Arnall T. A graphic language for touch-based interactions. In: Proceedings of the 8th conference on human-computer interaction with mobile devices and services. Helsinki, Finland: ACM; 2006.

[2] Bhattacherjee A. Understanding information systems continuance: an expectation-confirmation model. MIS Quarterly 2001;25(3):351-70.

[3] Bischoff-Ferrari HA, Vondechend M, Bellamy N, R.T. Validation and patient acceptance of a computer touch screen version of the WOMAC 3.1 osteoarthritis index. Annals of the Rheumatic Diseases 2005;64(1):80-4.

[4] Blake H. Innovation in practice: mobile phone technology in patient care. British journal of community nursing 2008;13(4). $160,162-165$.

[5] Bravo J, López-de-Ipiña D, Fuentes C, Hervás R, Peña R, Vergara $M$, et al. Enabling NFC technology for supporting chronic diseases: a proposal for alzheimer caregivers. In: Aarts E, Crowley J, De Ruyter B, Gerhäuser H, Pflaum A, Schmidt J, Wichert R, editors. In ambient intelligence. Berlin/Heidelberg: Springer; 2008. p. 109-25.

[6] Dale O, Hagen KB. Despite technical problems personal digital assistants outperform pen and paper when collecting patient diary data. Journal of Clinical Epidemiology 2007;60:8-17.

[7] Davis FD, Bagozzi RP, Warshaw PR. User acceptance of computer technology: a comparison of two theoretical models. Management Science 1989;35(8):982-1003.
[8] ECMA-340. Near Field Communication Interface and Protocol (NFCIP-1); 2004.

[9] Fikry, M, Karim A, Muhamad R. Integration of Near Field Communication (NFC) and Bluetooth technology for medical data acquisition system. Computational geometry and artificial vision; 2006. p. 147-52.

[10] Forum N. Smart poster record type definition. Available from: $\langle\mathrm{http}$ // www.nfc-forum.org/specs/ > [accessed 18.10.10].

[11] Grauer H, Birnbom F. A geriatric functional rating scale to determine the need for institutional care. Journal of the American Geriatrics Society 1975;23:472-6.

[12] Guyatt GH, Feeny DH, Patrick DL. Measuring health-related quality of life. Annals of Internal Medicine 1993;118(8):622-9.

[13] Hevner AR, March ST, Park J. Design science in information systems research. Management Information Systems Quarterly 2004;28(1):75-105.

[14] Iglesias R, Parra J, Cruces C, Segura NGD. Experiencing NFCbased touch for home healthcare. In: Proceedings of the 2 nd international conference on pervasive technologies related to assistive environments. Corfu, Greece: ACM; 2009.

[15] Java. What is J2ME or Java ME?; 2010. Available from: 〈http://www.java.com/en/download/faq/whatis_j2me.xml〉 [accessed 20.08.10].

[16] Jones D, Gregor S. The anatomy of a design theory. Journal of the Association for Information Systems 2007;8(5):312-35.

[17] Kasarskis EJ, Scarlata D, Hill R, Fuller C, Stambler N, Cedarbaum JM. A retrospective study of percutaneous endoscopic gastrostomy in ALS patients during the BDNF and CNTF trials. Journal of the Neurological Sciences 1999;169(1-2):118-25.

[18] Kaufmann P, Levy G, Thompson JLP, DelBene ML, Battista V, Gordon PH, et al. The ALSFRSr predicts survival time in an ALS clinic population. Neurology 2005;64:38-43.

[19] Koene P, Köbler F, Burgner P, Resatsch, F, Sandner U, Leimeister JM, et al. RFID-based media usage panels in supportive environments. In: Proceedings of the 18th European conference on information systems (ECIS); 2010.

[20] Lahtela A, Hassinen M, Jylha V. RFID and NFC in healthcare: Safety of hospitals medication care. In: Proceedings of the 2008 second international conference on pervasive computing technologies for healthcare; 2008.

[21] Leimeister JM, Krcmar H, Horsch A, Kuhn K. Mobile IT-Systeme im Gesundheitswesen, mobile Systeme für Patienten. HMD Praxis der Wirtschaftsinformatik 2005;41(244):74-85.

[22] Löser C, Lübbers H, Mahlke R, Lankisch PG. Der ungewollte Gewichtsverlust des alten Menschen. Deutsches Arzteblatt 2007;104(49):3411-20.

[23] Menschner P, Prinz A, Koene P, Köbler F, Altmann M, Krcmar H, et al. Reaching into patients' homes-participatory designed AAL services. Electronic Markets; 2011. p. 1-14.

[24] Morak J, Hayn D, Kastner P, Drobics M, Schreier G. Near field communication technology as the key for data acquisition in clinical research. In: Proceedings of the first international workshop on near field communication; 2009.

[25] Morak J, Kollmann A, Hayn D, Kastner P, Humer G, Schreier G. Improving telemonitoring of heart failure patients with NFC technology. In: Proceedings of the fifth IASTED international conference: biomedical engineering; 2007.

[26] Nyholm D, Kowalski J, Aquilonius S-M. Wireless real-time electronic data capture for self-assessment of motor function and quality of life in Parkinson's disease. Movement Disorders 2004;19(4):446-51.

[27] Palmblad M, Tiplady B. Electronic diaries and questionnaires: designing user interfaces that are easy for all patients to use. Quality of Life Research 2004;13:1199-207.

[28] Prinz A, Menschner P, Altmann M, Leimeister JM. inSERT-an NFC-based self-reporting questionnaire for patients with fine motor diseases. In: Proceedings of the 3rd international workshop on near field communication-NFC2011 Hagenberg; 2011. 
[29] Prinz A, Menschner P, Leimeister JM. NFC-basiertes Ernährungsmanagement für ältere, pflegebedürftige Menschen. Informatik 2009-Im Focus das Leben. Jahrestagung der Gesellschaft für Informatik. Lübeck: GI-Gesellschaft für Informatik. GI Lecture Notes in Informatics; 2009.

[30] Prinz A, Menschner P, Leimeister JM. Has NFC the potential to revolutionize self-reported electronic data capture?-an empirical comparison of different interaction concepts. In: Proceedings of the $\mathrm{CHI}$ conference on human factors in computing systems. Austin, USA: ACM; 2012.

[31] Richter JG, Nixdorf M, Koch T, Schneider M, Becker A, Monser R. Mobile computing instead of paper based documentation in German rheumatology. In: Proceedings of the international conference on mobile business IEEE computer society; 2006.

[32] Schweiger A, Sunyaev A, Leimeister JM, Krcmar H. Toward seamless healthcare with software agents. Communications of the Association for Information Systems (CAIS) 2007;19:692-709.

[33] Simon HA. The sciences of the artificial.3rd ed. Cambridge, MA: MIT Press; 1996.

[34] Strömgren AS, Groenvold M, Sorensen A, Andersen L. Symptom recognition in advanced cancer. A comparison of nursing records against patient self-rating. Acta Anaesthesiologica Scandinavica 2001;45(9):1080-5.
[35] Subramanya SR, Yi BK. User interfaces for mobile content. IEEE Computer 2006;39(4):85-7.

[36] Velikova G, Wright EP, Smith AB, Cull A, Gould A, Forman D, et al. Automated collection of quality-of-life data: a comparison of paper and computer touch-screen questionnaires. Journal of Clinical Oncology 1999;17(3):998-1007.

[37] Want R. An introduction to RFID technology pervasive computing. IEEE 2006;6:25-33.

[38] Welsh M, McDermott MP, Holloway RG, Plumb S, Pfeiffer R, Hubble J. Development and testing of the Parkinson's disease quality of life scale. Movement Disorders 2003;18(6):637-45.

[39] Wolfe F, Pincus T. Data collection in the clinic. Rheumatic Disease Clinics of North America 1995;21(2):321-58.

[40] Leimeister JM, Daum M, Krcmar H. Towards mobile communities for cancer patients: the case of krebsgemeinschaft.de. International Journal of Web Based Communities (IJWBC) 2004;1:58-70.

[41] Leimeister. Service Design Dienstleistungsengineering und management: Berlin Heidelberg: Springer 2012. p. 159-187 http://dx.doi.org/10.1007/978-3-642-27983-6_5. 